\title{
Le Genre en faces
}

Variations autour de deux paradigmes

Nicolas Adell

\section{(2) OpenEdition} Journals

Édition électronique

URL : http://journals.openedition.org/ress/1055

DOI : $10.4000 /$ ress. 1055

ISSN : 1663-4446

Éditeur

Librairie Droz

\section{Édition imprimée}

Date de publication : 15 décembre 2011

Pagination : 231-257

ISBN : 978-2-600-01575-2

ISSN : 0048-8046

\section{Référence électronique}

Nicolas Adell, «Le Genre en faces », Revue européenne des sciences sociales [En ligne], 49-2 | 2011, mis en ligne le 01 janvier 2015, consulté le 23 avril 2019. URL : http://journals.openedition.org/ress/1055; DOI : 10.4000/ress. 1055 


\title{
LE GENRE EN FACES \\ VARIATIONS AUTOUR DE DEUX PARADIGMES
}

\author{
NICOLAS ADELL \\ Université de Toulouse-II - LISST \\ nicolas.adell@univ-tlse2.fr
}

\begin{abstract}
Résumé. À partir d'une enquête ethnographique et historique menée auprès de communautés initiatiques d'artisans, les compagnons du Tour de France, l'auteur avance l'hypothèse que l'attention que ces communautés ont porté au genre est le reflet d'un rapport au genre particulièrement développé dans les sociétés occidentales entre la fin du $X \mathrm{~V} \| I^{\mathrm{e}}$ siècle et le milieu du $X \mathrm{X}^{\mathrm{e}}$ siècle. Ce paradigme est qualifié de masque en raison de l'importance qu'il accorde à la question des rôles de genre, à l'idée de sexe social, postures qui dessinent, en creux, une naturalisation inédite du genre et cristallise la bicatégorisation des sexes. À l'opposé de cette attitude, l'auteur décrit une autre modalité d'appréhension du genre, le paradigme visage. Celui-ci, que l'on retrouve à l'œuvre dans certaines sociétés extra-occidentales ainsi que dans une certaine avant-garde des sociétés européennes actuelles, fournit les ressources pour penser un genre en mouvement, non fondé sur la nature (du corps ou de l'esprit), mais établi sur des critères fondamentalement relationnels.
\end{abstract}

Mots-clés: théorie du genre, anthropologie des sexes, rôles sexués, compagnonnage

\begin{abstract}
From a historical and ethnographic study conducted among initiation groups of craftsmen, compagnons du Tour de France, the author speculates that the attention that these communities have focused on gender is a reflection of a specific relation to gender developed in Western societies from the late eighteenth to the mid-twentieth century. This paradigm is called mask because of the importance it attaches to the issue of gender roles, to the idea of social sex. That attitudes draw, by contrast, an unprecedented naturalization of gender and crystallizes sex-duality. In contrast to this posture, the author describes another kind of gender conception, the paradigm face. This one, which is at work in some non-Western societies as well as in the avant-garde of contemporary European societies, provides the resources to think about gender in motion, not based on the nature (of the body or mind), but on fundamentally relational criteria.
\end{abstract}

Keywords: gender theory, genderstudies, anthropology of the sex roles, compagnonnage 


\section{INTRODUCTION Deux paradigmes}

L'objectif de ce texte est de montrer, d'une façon parfaitement exploratoire, que l'un des effets ignorés de cette période de profondes transformations qui s'étend entre le $\mathrm{XVIII}^{\mathrm{e}}$ et le début du $\mathrm{Xx}^{\mathrm{e}}$ siècle en Europe consiste en un changement de paradigme concernant l'appréhension du genre des personnes qui intègre et dépasse largement l'invention de l'idée du «sexe social». Cette exploration sera volontiers diachronique et comparative afin de restituer le mouvement général de cette conversion du rapport au genre, mais elle s’appuiera également sur les données précises empruntées à un dossier que j’ai ouvert depuis quelques années, celui des compagnons du Tour de France. Il s’agit d’artisans de haut niveau regroupés en communautés initiatiques et dont la formation consiste notamment en une période d'itinérance (de 3 à ıo ans) dite «Tour de France »'.

Le compagnonnage présente en effet un certain nombre de caractéristiques qui le rendent particulièrement intéressant pour qui cherche à observer les enjeux, modalités et formes de la production du genre dans ces sociétés de la modernité européenne. Institution largement issue de cette période de grandes transformations, en dépit du caractère apparemment suranné de certaines pratiques (initiations, rites de reconnaissances, etc.), le compagnonnage est un groupe qui pense particulièrement sa reproduction sociale, sa continuité, et explore cette pensée dans toutes les voies qu'elle lui propose: initiatique, pédagogique, coutumière, réglementaire. Cette reproduction sociale comprend également une reproduction de genre : il s'agit d’une institution qui s'est nettement affirmée comme responsable de la production d’un idéal masculin dans le monde artisanal. Et cet accès à la virilité a longtemps formé le cœur du dispositif compagnonnique².

I Un ouvrage synthétisant les principaux résultats de mes enquêtes sur ces communautés compagnonniques a paru récemment: N. Adell (2008).

2 Pour plus détails, voir N. Adell (ibid, p. 110-125). 
D'où la seconde raison qui fait du compagnonnage une entrée efficace pour questionner le genre. En effet, depuis peu (2004) mais après plus de trente ans de débats, certains groupes de compagnons ont fini par admettre d'initier des femmes. Il se joue ainsi sous nos yeux un moment critique propice à l'examen des rapports entre les sexes, à l'observation de la saisie par les compagnons du genre qui, brusquement, devient une catégorie qu'il s'agit de repenser entièrement et qui acquiert de ce fait une nouvelle pertinence. Comment une institution qui produit du masculin et qui, de surcroît, pense cette production, peut-elle composer avec l'entrée en mixité, avec cette mise en mouvement du genre qui était jusqu’à présent un donné évident et univoque que des régularités et des coutumes de métier et d'initiation avaient progressivement associé et enraciné dans des pratiques, des lexiques, des habitudes, en faisant ainsi une sorte d'actif invisible? Les enquêtes que j’ai conduites entre 2000 et 2006 parmi plusieurs groupes de compagnons en France ${ }^{3}$, et plus spécifiquement auprès d'une centaine de charpentiers à Paris, Marseille et Toulouse, ont permis de dégager les ressources de l'identité de genre qui y est façonnée. Ce travail est actuellement poursuivi avec l'aide d'une collaboratrice, Liliane Hilbrandt, dans une volonté de saisir, depuis l'irruption des initiations de femmes, ce que le féminin fait au compagnonnage.

L'exposé des résultats de cette enquête ethno-historique servira d’amorce, la complexité du dossier compagnonnique nécessitant une présentation spécifique, pour proposer, dans un second temps, un tableau des formes et des ressorts du changement de paradigme observé. Celui-ci consiste en une opération qui, progressivement, rend le genre «visible», qui en fait un objet de réflexion en soi et non plus seulement une catégorie sous-jacente aux représentations et aux pratiques. Non que le genre et ses expressions fussent auparavant cachés et ce dans toutes les sociétés où se distinguent, au moins, le féminin et le masculin dans l'exercice de certaines tâches, l'accès à certains savoirs, ou l'économie des

3 Les quelques 20000 compagnons que l'on trouve aujourd'hui en France sont répartis en différentes communautés. Les plus importantes sont l'Association ouvrière des Compagnons du Devoir du Tour de France, la Fédération compagnonnique des Métiers du Bâtiment et autres activités, et l'Union compagnonnique des Devoirs Unis. 
émotions. Simplement, il acquiert dans la modernité européenne une visibilité superlative, fonctionnant comme une super-catégorie (tout devient susceptible d'être dit dans le langage du genre) qui contribue, paradoxalement, à en rigidifier les formes (le féminin et le masculin s'excluent de plus en plus radicalement). Aussi, pour exprimer tout à la fois ce plus de visibilité et cet effet de fixation, j’ai nommé ce paradigme «moderne» le masque, tandis que l'autre, plus mobile, mais moins détaché, sera qualifié de visage. Et c’est à la caractérisation de ce genre-masque et de ce genre-visage que cet essai sera finalement consacré.

\section{LE GENRE EN COMPAGNONNAGE}

\section{I.I. NAISSANCE DU GENRE \\ La division sexuée de l'ordre compagnonnique}

Ensemble disparate, le compagnonnage s'est longtemps caractérisé essentiellement par la jeunesse et l'itinérance de ses membres. Être compagnon, jusquau début du XIX siècle, c'était être sur les routes, changer de boutique régulièrement; c'était également faire des «niches», des tours, aux ouvriers sédentaires, aux Anciens installés. Le compagnonnage avait alors quelque chose des organisations de jeunesse de l'Ancien Régime. De ce fait, il encourageait l'exercice d'une sociabilité masculine et favorisait le passage à l'âge adulte pour les hommes, même si, bien évidemment, il n'était pas pensé sous cet aspect. Mais, lors du remaniement profond de l'organisation compagnonnique qui a eu lieu au tournant des XVIII et XIX siècles, effet des transformations (sociales, économiques, politiques, etc.) plus générales du moment, le genre fait son apparition comme enjeu de plus en plus explicite de la formation compagnonnique. Précisément, c'est ce genre-masque qui se met lentement en place, à savoir, une approche qui radicalise le dimorphisme sexuel, l'approfondit et installe avec force l'idée que ce qui est compagnonnique est viril.

Au cours du XIX siècle, les rituels d'initiation compagnonniques, notamment celui dit de Réception qui fait le compagnon, se chargent d'une dimension physique importante rendant le corps mâle seul apte à être initié. Si, en raison du secret qui entoure ces rites, l'on n'en possède que peu de descriptions précises, les rares témoignages que nous ayons pour la fin du XIX siècle, concernant les char- 
pentiers, attestent leur violence et l'importance accordée à la virilité dans tous ses états ${ }^{4}$; la dimension sexuelle de l'initiation y occupe une place considérable. Mieux encore, la Réception est pensée comme le moment où s'acquiert cette qualité proprement virile qui est la «maîtrise de soi» et dont les compagnons, charpentiers d’abord, ont proposé la traduction dans leur lexique spécifique: l'Orient ${ }^{5}$. Avoir de l'Orient, c'est, sur un chantier et dans la vie, savoir s'orienter, anticiper les difficultés, se sortir d’une situation délicate, être ingénieux. C’est faire preuve, comme disaient les Grecs, de mètis (Detienne M., Vernant J.P., 1978).

Mais ce souci viril du compagnonnage, cette prise de conscience du genre comme qualité du compagnon, apparaît avec le plus de force, paradoxalement, dans les rôles neufs qui sont accordés aux femmes dans l'organisation compagnonnique et autour d'elle. En somme, deux figures principales se partagent l'horizon féminin de l'homme en compagnonnage à partir du XIX siècle : la Mère et la bonne amie. L'une comme l'autre ont pu exister auparavant, incarnées dans des personnages singuliers dont on peut trouver, rarement cependant, la trace dans les vies de quelques compagnons. Néanmoins, ce n’est que dans le cadre de la restructuration profonde du monde compagnonnique qu'elles accèdent véritablement au niveau de types sociaux, s'idéalisant dans de pures fonctions et étant véritablement prises en charge de manière compagnonnique. Ainsi, la figure de la Mère, femme qui héberge et gère la vie matérielle des itinérants et dont la fonction s'est perpétuée jusqu'à nos jours (avec toutefois un poids relatif selon les groupements et les corps de métier considérés), devient centrale à partir des années I820. Jusque-là, elle jouait un simple rôle de logeuse, en compagnie de son mari, liée aux compagnons par contrat; désormais, elle accède à davantage de secrets, fait l'objet d'une initiation spéciale (la Réception de Mère) et obtient même, pour certains métiers, un statut doté d'une sacralité inédite en compagnonnage. Ainsi, dans les années I820, la Mère était dite «sacrée» chez les forgerons; aujourd’hui

4 II faut noter que le témoin (J. Connay, 1909) est également un dénonciateur qui cherche à régler des comptes avec sa société compagnonnique. Témoignant à charge, sa description n'en contient pas moins un canevas dont des échos contemporains attestent qu'il n'est pas si étranger aux pratiques actuelles. Pour une présentation et une analyse de ce rituel, voir N. Adell (2008, p. I8I-187).

5 Sur cette notion, voir N. Adell (2004). Et sur la maîtrise de soi chez les garçons, lire les pages qu'y consacre A.-M. Sohn (2009, p. 18-40 et 93-98). 
encore, les charpentiers de la Fédération et de l'Association ne s'adressent à cette personne qu'en lui disant «Notre Mère» en une sorte de révérence toute religieuse. Cette idéalisation de la Mère engage plusieurs conséquences: la volonté de faire communauté sur le modèle de la famille, l'intégration des femmes dans le système compagnonnique des valeurs et des rôles, et, en négatif, la dimension masculine de l'exercice compagnonnique. Les nécessités de la vie sociale obéissent, selon les compagnons, à un partage entre les sexes que le compagnonnage a rigidifié. En intégrant les femmes dans des rôles limités et précis, aussi importants qu'ils puissent être, les compagnons les excluaient dans le même temps et plus explicitement sans doute quauparavant de leur monde privé. On rend manifeste leur impossibilité d'être «compagnonnes », d'accéder à certains secrets, d'investir les métiers, tous ces domaines qui sont de plus en plus vécus comme coextensifs à l'affirmation d'une virilité dont on prend peu à peu conscience.

De plus, cette intégration s'est doublée de la mise en place, implicite, d’une hiérarchie des genres. Celle-ci ne se traduit nulle part ailleurs mieux quau travers des initiations respectives des compagnons et de la Mère. Dans les rares corps de métier pour lesquels nous possédons des descriptions assez précises des rituels de réception de Mère et de compagnons, les blanchers-chamoiseurs au milieu du XIX ${ }^{\mathrm{e}}$ siècle par exemple, il est possible d'observer à quel point le rituel concernant la Mère est considéré comme une version volontairement dégradée du rite qui fait le compagnon (N. Adell, 2006a, p. 535-54I).

Le sentiment se fait croissant chez les compagnons au tournant des XVIII et $\mathrm{XIX}^{\mathrm{e}}$ siècles que les individus sont dotés d'une nature véritable qu'il s’agit de révéler par divers moyens dont le plus grandiose est l'initiation. L'importance qui est attachée à la délivrance du nom d'initié à chaque récipiendaire en constitue une preuve déterminante. Ce nouveau nom obéit à un ensemble de règles qui, en dépit de menues variations selon les corps de métier, sont relativement stables pour l'ensemble des compagnonnages. Ce nom compagnonnique est formé d'une part d'un terme évoquant l'origine géographique de la personne, d’autre part d'une épithète évoquant une qualité (ou, plus anciennement, une particularité qui pouvait navoir rien d'enviable, à la manière des qualificatifs royaux : le Borgne, etc.) d'ordre technique, moral ou physique. On trouve ainsi chez les compagnons des Béarnais la 
Prudence, des La Sagesse de Tours, des Angevin l'Ami des Filles, des Lyonnais le Bon Cour, des Parisien la Constance. Le nom compagnonnique ne traduit pas de ce fait une personne entièrement neuve. En réalité, il exprime la nature d'un individu (comme on exprime le jus d'une orange), en fait ressortir les traits saillants et distinctifs. Le traitement du genre obéira à la même logique; considéré en tant qu'élément d'une «nature» humaine, il nécessitera désormais d'être signifié, comme la provenance géographique ou les qualités morales dans le nom, à la faveur de moments, d’actes ou de gestes singuliers tels que l'initiation, l'apprentissage du métier, la vie en communauté et, bien évidemment, les relations amoureuses.

Ce qui nous conduit à évoquer l'autre image qui, à partir du XIX ${ }^{e}$ siècle, occupe l'espace des représentations compagnonniques de la femme: la bonne amie. J’ai pu montrer ailleurs comment s'opérait, entre la fin du XVIII et le début du XIX ${ }^{e}$ siècle, une rupture dans les discours concernant les rapports amoureux et la sexualité avec le passage d'un discours sur le sexe intégré dans des énoncés plus généraux sur le jeu et l'amusement à un discours sur le sexe qui rejoint les énoncés sur l'altéritét. La transition de l'un à l'autre semble hautement significative de la naissance du genre au sein du compagnonnage, et précisément de ce genre-masque déclinant des identités que marquent des attributs qui sont censés trahir une nature (et plus tard, mais le fondement reste le même, la travestir). Désormais inscrite sous l'espèce du Différent, le féminin pourra servir à penser, en négatif, le Même comme masculin. Ainsi, parmi les expressions typiques de la différence chez les compagnons figure le thème de la bonne amie qui attend le retour de l'itinérant qui poursuit son Tour de France: les lithographies compagnonniques, les chansons, les autobiographies ne se lassent pas d'évoquer la femme à laquelle on demande patience et fidélité ou, en cas de refus, de relater la victoire du Devoir sur l'Amour (et dont l'ethnographie tend à relativiser fortement le caractère systématique). La maîtrise de soi, cette démonstration permanente de virilité pour les compagnons, s'exprime d’abord dans la capacité que l'on a de suivre une ligne de conduite que, mieux que toute autre voie, le Tour de France matérialise. L'achever, c'est déjà se conduire en homme. 


\section{I.2. LA FÉMINISATION DU COMPAGNONNAGE Problèmes et enjeux}

Si la prise en considération des femmes dans la sphère compagnonnique, dans son lexique, dans ses rites et dans ses valeurs, a signifié dans un premier temps une prise de conscience de la dimension masculine de l'existence compagnonnique (être compagnon, c'est être un homme d'abord), un niveau supérieur d'intégration, comme celui qui permettrait aux femmes d'intégrer le processus de formation, le Tour de France et d'être reçues comme compagnon (ou «compagnonne», la question se pose toujours), promettait d'aboutir, c'est en tout cas le destin tragique que beaucoup prédisaient, à une remise en question de l'identité compagnonnique tant au niveau collectif qu'au niveau singulier. Qu'est-ce qu'être compagnon si les femmes peuvent en être?

Mais il existe, avant cette extrémité que tous les compagnonnages n'ont pas atteinte, des degrés intermédiaires qui manifestent l'évolution du traitement réservé aux femmes et, plus généralement, du rapport au genre en compagnonnage. Cela se perçoit notamment dans le sort qui est réservé aux épouses de compagnons. Celles-ci, invisibles ou presque durant un long $\mathrm{XIX}^{\mathrm{e}}$ siècle (à l'exception de celles qui sont «Mères »), font, au début du $\mathrm{xx}^{\mathrm{e}}$ siècle, leur entrée dans le monde compagnonnique par l'intermédiaire des banquets organisés à l'occasion des fêtes patronales des corps de métier, qui sont un moment central de la vie compagnonnique. Dans les années 1930, plusieurs corporations ont commencé à admettre les épouses à leur table. D’après Laurent Bastard, l'Union compagnonnique, dont des principes d'ouverture et de «progrès » ont présidé à la fondation en I889, semble avoir été la première à admettre les femmes aux banquets de fêtes patronales (Bastard L., 2004, p. 35).

Mais cela ne signifie pas que les épouses de compagnons sont, à partir des années 1930, considérées sur un pied d'égalité par rapport à leur mari, loin de là. Il ne faut pas non plus y chercher l'origine lointaine de la féminisation actuelle de certains compagnonnages. Cette présence féminine au banquet signale simplement, mais c'est déjà beaucoup, un nouveau statut de la femme chez les compagnons qui la rapproche du cercle compagnonnique privé. En effet, vers la fin du $\mathrm{XIX}^{\mathrm{e}}$ siècle et le début du $\mathrm{Xx}^{\mathrm{e}}$ siècle, le mariage n’apparaît 
plus comme une fin de jeunesse et de compagnonnage (comme ce pouvait être le cas dans l'ancienne formule car le mariage empêchait l'itinérance), le moyen d'une délivrance des liens fraternels, mais comme un critère qui établit et valide ce nouveau statut qu'est celui de compagnon sédentaire. L'épouse a ainsi un rôle à jouer dans la formation de l'être compagnon, un rôle qu'on lui reconnaît «officiellement» par sa présence au banquet «privé » : elle est cet attribut nécessaire à l'accomplissement de son destin d'homme chez les compagnons. Mais le siège compagnonnique reste fondamentalement une maison-des-hommes.

Cette dimension s'est trouvée particulièrement renforcée ces dernières années par le biais des nouveaux réseaux sociaux. Il existe à l'heure actuelle un forum consacré aux «compagnonnettes » (tel est le terme par lequel elles s'autodésignent), ces petites amies, épouses, copines d'aspirants ou de compagnons et par l'intermédiaire duquel des femmes, jeunes souvent, partagent les plaisirs, les joies, les peines, les difficultés qu'elles éprouvent dans la vie de tous les jours avec leur partenaire itinérant, attaché à une communauté particulière ${ }^{7}$. Il s'y manifeste une identité de genre forte, «traditionnelle» dans le sens où elles s'inscrivent dans cette longue lignée de figures féminines qui, depuis la bonne amie et en passant par l'épouse admise au banquet, parcourt l'histoire compagnonnique. D’une certaine manière, cette identité prend ici une consistance qu'elle n'avait pas auparavant, nébuleuse d'individualités, par le biais de cet espace réservé qui donne véritablement corps au groupe des «femmes de compagnons». La page d’accueil du forum précise, à l'attention des compagnons, l'intention collective et identitaire (façonnée en miroir de l'identité compagnonnique : le forum s'intitule, «Notre corpo à nous ») qui y est en jeu : «Passe ton chemin s'il te plaît... nous respectons vos traditions... respecte ce forum».

Une des probables raisons qui font que ce motif s'est renforcé ces dernières années est précisément le surgissement d’une autre figure, inédite, celle de la femme-compagnon, qui brouille les anciennes catégories. En effet, comme bien d'autres communautés ou groupes professionnels, le compagnonnage a été confronté dans la seconde moitié du $\mathrm{xx}^{\mathrm{e}}$ siècle, et avec une acuité plus forte à 
partir des années 1980, à ce que l'on appelait alors (et que l'on appelle toujours d'ailleurs chez les compagnons) la «question des femmes ». Cette «question», posée à tout groupe masculin de fait (l’armée, la police, le compagnonnage, la légion étrangère, etc.), est la suivante : l'intégration des femmes entraînera-telle une déformation relativement négligeable de l'institution ou en ruinera-telle les fondements? Dans ce dernier cas, sera-ce un arrêt de mort définitif ou bien l'occasion d'une nécessaire régénération? Le cas de la police, récemment étudié par Geneviève Pruvost, a permis à l'ethnologue de conclure que, pour ce groupe précis, «la féminisation [...] ne transforme pas fondamentalement l'institution» (Pruvost G., 2007, p. 238). Elle montre clairement, à l'aide d'une riche ethnographie, que les valeurs et les pratiques attachées à la virilité traditionnelle (force, courage, violence [ibid., p. 217-218]) ainsi que la puissance de l'hétéronormativité ne sont en rien affectées par l'intégration des femmes dans la police (ibid., p. 249). Mieux, la mise en œuvre de ces usages par des femmes est toujours susceptible de se retourner contre elles : la brutalité féminine peut être le fait d'une hystérie plus ou moins latente (ibid., p. 232). Il est difficile d'aboutir, pour le moment, à un tel constat chez les compagnons le phénomène de féminisation étant plus récent et plus partiel.

Deux groupements sur les trois, la Fédération et l'Association, ont accepté il y a une dizaine d'années que les filles suivent leur formule d'apprentissage pour les premières étapes, jusqu'au moment de la première initiation, l'Adoption, qui signifie le départ sur le Tour de France. La moindre importance accordée par l'Union à ce temps d'apprentissage ne lui a pas offert l'opportunité de réaliser cette intégration-test. Sans doute également, ce dernier groupement, en tant qu'il est celui qui s'est le plus ouvert à la diversité des corps de métier et a le premier évoqué la possibilité d’une présence des épouses lors des banquets corporatifs, s'est appliqué plus que les autres au respect d’autres usages comme celui d'un recrutement strictement masculin.

Puis, en 2004, l'Association a décidé d'admettre les filles sur le Tour de France. Ainsi, au début de l'été 2005, six jeunes filles étaient adoptées: quatre en taille de pierre, une dans la tapisserie, une pour exercer le métier de peintre en bâtiment. Mais l'événement décisif, celui qui, au sein même du groupement, lors 
même de la cérémonie initiatique, a soulevé le plus de discussions, s'est déroulé le 18 novembre 2006, à Saumur, où il a été procédé à la Réception de Lucie Branco, dite La Sincérité de Lille, compagnon tailleur de pierre. La terminologie est encore flottante. Dans un premier temps, le terme de «compagnonne» avait la préférence; mais il semble désormais entendu de parler de «compagnon» pour ces femmes reçues. Ce choix paraît significatif d’une volonté, historique, de déconnecter le genre de la formule compagnonnique pour le laisser dans des espaces séparés (le forum des «compagnonnettes» par exemple). Le compagnonnage, sous ce nouvel aspect qui nous semble constituer une rupture d'un niveau équivalent à celle qui s'est produite au tournant des XVIII et XIX ${ }^{\mathrm{e}}$ siècles, ne proposerait plus de former des hommes mais des personnes. C'est une nouvelle ambition. Toutefois, le recul que nous avons sur l'événement ne permet pas de formuler plus que de simples conjectures dont quelques indices tout récents laissent promettre qu'elles pourront servir d'hypothèses de travail.

Le genre-masque, celui qui caractérise le compagnonnage moderne et sa mise au point des rôles masculins et féminins, n'est pas un genre que l'on porte à sa guise et dont on peut entièrement choisir la teneur. Il s'agit bien plutôt d'une conception qui atteste une profondeur inédite du genre, car il sous-entend l'idée d'un genre «véritable», «naturel» que le masque affiche ou dissimule (c'est l'ambivalence même de tout masque). Si le genre-masque soulève la possibilité du travestissement, il ne l'encourage pas pour autant. Bien au contraire, ayant pris conscience de l'importance de la dimension construite du genre, j’y reviendrai, les tenants du genre-masque, dont les compagnons font partie (le compagnonnage moderne fabrique du masculin), se sont appliqués à valoriser la transparence du masque, son adéquation avec le genre «véritable» qui, pour les compagnons (et ils sont loin d'être les seuls dans ce cas), est le sexe biologique. Ainsi, parmi les quelques femmes qui font leur Tour de France (il y en a une trentaine à l'heure actuelle) et celles qui sont compagnons, les membres de l'Association marquent, vis-à-vis du public et de l'ethnologue, leur préférence pour celles qui sont «féminines»: mariées ou en couple, maquillées (discrètement), dans des habits que des hommes ne peuvent porter selon les standards de la culture européenne. Telle jeune fille, apprentie cuisinière, que je rencontrai dans un siège 
compagnonnique parisien en 2010, avait suscité de la part des compagnons qui m'entouraient les commentaires suivants: «Tu vois, elle, avec sa gitane maïs, sa dégaine, la façon dont elle parle... On ne peut pas dire qu'elle représente le mieux l'intégration des femmes!» (compagnon mécanicien). Le type du garçon manqué, fréquent cependant tant les filles cherchent à saffirmer et à ajouter même aux usages dits masculins un plus qui compense leur «déficit» physiologique, n'est pas celui que recherchent les compagnons qui, sur ce point, semblent se distinguer des policiers étudiés par Geneviève Pruvost.

L'unisexe, fondé sur un modèle masculin, n’est pas nécessairement ce à quoi le compagnonnage veut aboutir. D’ailleurs, cette orientation des sociétés contemporaines vers l'unisexe, que pensent observer plusieurs chercheurs dans l'examen des tenues vestimentaires, des usages des loisirs, de la mode en général, me semble refléter davantage une insuffisance descriptive qu'une réalité de premier ordre. Le dimorphisme sexuel se maintient à une autre échelle, dans d’autres objets et dans d’autres détails que ceux auxquels nous avons été habitués et qui, pour être plus discrets et parfois indiciels, n'en constituent pas moins des écarts dotés d'une puissance signifiante importante. Il reste que l'on ne passe sans doute pas aisément d'une société masculine comme les compagnons, mieux, vouée à la construction du masculin, à une société qui ne connaîtrait que des personnes, indifférente en apparence à leur sexe. Les résistances des autres groupements le prouvent. De plus, l'on aura sans doute l'occasion d'observer, au moins dans un premier temps (dont on ne saurait présumer l'étendue), les manières dont se pense et s'élabore l'identité masculine chez les compagnons avec, peut-être, l'émergence d'une «pensée de la différence» entre masculinité et virilité telle que celle-ci a pu être constatée au sein de l'armée ou dans le monde ouvrier à partir de la psychologie du travail ${ }^{8}$. 


\section{LE VISAGE ET LE MASQUE}

L'irruption du genre en compagnonnage, la dimension centrale qu'il acquiert dans l'institution et les problèmes inédits qu'il pose dans l'épreuve de la mixité, me semble constituer des caractéristiques d'une approche du genre spécifiquement «moderne» (entendons par là, «propre aux sociétés occidentales entre la fin du $\mathrm{XVIII}^{\mathrm{e}}$ siècle et le milieu du $\mathrm{xx}^{\mathrm{e}}$ siècle »), ce que je qualifie le paradigme du masque. Précisons cependant que, dans cette querelle des paradigmes que je me propose d'exposer ici, il serait gravement préjudiciable de considérer qu'elle s'épuise dans une chronologie qui verrait purement et simplement un paradigme succéder à l'autre. Comme à l'ordinaire, les choses sont à nuancer et l'on verra que, sous l'emprise temporaire de l'un, se dissimule et parfois réalise des percées décisives l'autre saisissement du genre.

\section{I. LE PARADIGME VISAGE \\ Le genre en miettes}

Pour autant qu'il est à mes yeux celui qui nous est à l'heure actuelle le plus «étranger» (on a reconnu, dans le compagnonnage, la familiarité du masque), le paradigme visage est celui qui, dans le cadre de cette approche générale, me semble devoir être caractérisé le premier. Trois éléments distinctifs y sont à l’œuvre : l'idée de surface, la possibilité d'une lecture des signes du genre, l'existence d'un monde tout en continuité. Expliquons chacun de ces traits afin d'en mesurer l'impact.

Dans le genre-visage, l'on trouve l'idée, ou la croyance, que l'identification du genre d'un individu occupe un espace essentiellement épidermique. C’est, si l'on veut, l'immédiateté du genre. Le féminin comme le masculin se donnent à voir sur les êtres, humains ou pas, selon des qualités qui sont d'abord des apparences. De ce point de vue, le genre est essentiellement «dit»: il est de l'ordre du langage, que celui-ci soit la parole ou tout autre système signifiant (les attitudes corporelles, les vêtements, etc.). Il relève d'une «adresse» qui a pour destinataire l'environnement social devant qui le genre est étalé. Mieux cet étalement épuise le genre qui n’a absolument, selon cette perspective, aucune épaisseur. Il est sans ancrage. C'est, semble-t-il, l'une des leçons essentielles à tirer des travaux de Thomas Laqueur (1992), que poursuivent et confortent, pour la période contem- 
poraine, ceux d'Alice Dreger (1998) sur le traitement des hermaphrodites. Si, pour résumer brièvement les conclusions de Laqueur, le genre existe avant le sexe, c'est que, précisément, le genre n’a pas, jusqu’à une époque relativement récente, de véritable profondeur puisque, si l'on cherche à l'intérieur des corps, si on les fouille comme en ces débuts de systématisation de l'anatomie', T. Laqueur montre bien que l'on ne découvre qu'un sexe unique. Saurait-on mieux exprimer la continuité du monde autrement que dans cette indétermination originelle? J’y reviendrai.

Or, si le genre ne se déploie qu'en surface, c'est donc, deuxième trait, qu'il est justiciable d'une lecture totale, qu'il se dit entièrement dans les signes qui le portent et le font mais qui varient selon les sociétés qui ont chacune leur lexique du genre. Ainsi, le genre est une information à laquelle on peut tenir, à laquelle on peut être tenu, mais dont on pourrait également se passer. De ce point de vue, une prolongation contemporaine et radicale du paradigme visage réside dans la vision de la personne exprimée par Antonin Artaud. La personne est d'abord un corps, et un corps dont la réalisation la plus parfaite est de s'épuiser, physiquement et symboliquement, dans des actes afin de sortir $\mathrm{du}$ «cadastre anatomique du corps présent $\gg^{10}$. La lecture affirme ici ses droits avec beaucoup de force dans la mesure où elle ne réclame que d'être élémentaire: l'individu ne se définit que dans une pragmatique dont son corps est le principal acteur et dont aucune détermination ne doit entraver la liberté. C'est toute l'idée d'un «corps sans organes» avancée par A. Artaud (2004b, p. I653) et qui sera reprise par G. Deleuze et F. Guattari (1972, 1980) en la détournant quelque peu. Ce «corps sans organes» peut être compris et analysé de différentes manières en tant que corps sans contraintes, en tant que pur devenir par exemple. Du point de vue qui nous intéresse ici, celui du genre, il nous amène vers l'affirmation d'un corps sexuel l'emportant sur un corps sexué. Le paradigme visage, considéré dans ses extrémités, abolit le genre en pointant justement son absence de profondeur. Cette caractéristique, significative du paradigme visage, Artaud l’a bien vue, lui qui écrit à Rodez en mars 1946 :

9 Parmi une riche bibliographie, on pourra consulter les travaux d'Andrea Carlino (1994).

10 Cité dans E. Grossman (2004, p. 17). 
La réalité n’est pas dans la physiologie du corps mais dans la perpétuelle recherche d'une incarnation qui, perpétuellement désirée par le corps, n’est pas de chair mais d'une matière qui ne soit pas vue par l'esprit ni perçue par la conscience et soit un être entier de peinture, de théâtre et d'harmonie (Artaud A., 2004a, p. 1065).

Cette idée sera reprise, avec d'autres termes et dans une perspective plus militante, une trentaine d'années plus tard dans le célèbre article que Gayle Rubin a consacré à «l'économie politique du sexe » où elle indique, après une critique des conceptions marxistes et structuralistes du genre, que:

Nous ne sommes pas seulement opprimées en tant que femmes, nous sommes opprimées par le fait de devoir être des femmes ou des hommes selon le cas. Mon sentiment personnel est que le mouvement féministe doit rêver à bien plus encore qu'à l'élimination de l'oppression des femmes. Il doit rêver à l'élimination des sexualités obligatoires et des rôles de sexe. Le rêve qui me semble le plus attachant est celui d'une société androgyne et sans genre (mais pas sans sexe) où l'anatomie sexuelle n'aurait rien à voir avec qui l'on est, ce que l'on fait, ni avec qui on fait l'amour. (Rubin G., 1998 [1975], p. 64).

Enfin, si le genre-visage s'exprime en surface et qu'il se soumet dès lors à la possibilité d'une lecture, c'est qu'il relève d'un monde à dimension unique, un monde de la continuité du genre où tous les «faits» sont de même niveau et où ce qui les institue n'est pas tant une essence mais bien «l'action» (pour reprendre Artaud), autrement dit l'ensemble des relations qu'ils entretiennent les uns avec les autres. Dans cette perspective, le genre s'affirme fondamentalement comme un rapport et son mode d'être est essentiellement relationnel ainsi que le rappelle avec force Irène Théry dans un texte récent (2009, p. 17-25) qui s'appuie en particulier sur les analyses décisives de Marilyn Strathern (1988) menées à partir d'une riche ethnographie des Hagen vivant sur les Hautes-Terres de Nouvelle-Guinée. $\mathrm{Si}$, dans son extrémisme, le paradigme visage requiert l'abolition du genre, c'est qu'il prend conscience dans une certaine mesure de la mobilité du genre, de l'existence d'une ligne de flottaison où il se recompose perpétuellement selon les mouvements des éléments qui le constituent et dont la permanence, voire l’apparente stabilité, n'est que le fait d'une optique paresseuse ou imprécise ${ }^{11}$. 
C'est bien ce qui est en filigrane au travers de la croyance qu'étudie T. Laqueur à propos de l'androgynie originelle : la continuité du monde se lit dans la continuité des sexes (la femme est un homme non «déployé», avec le sexe «en dedans »). Ainsi, alors même que les sexes manifestent fondamentalement le principe de toute disjonction, s'il se trouve entre eux un ordre qui rend possible le passage de l'un à l'autre, c'est le monde entier qui se place sous le signe de l'unique et du continu. Une anecdote rapportée par Montaigne au cours d'un voyage fait à Vitry-le-François illustre parfaitement cette conception.

Passant à Vitry-le-François, je pus voir un homme que l'évêque de Soissons avait nommé Germain en confirmation [de son état] et que tous les habitants du lieu ont vu et connu fille jusqu'à l'âge de vingt-deux ans sous le nom de Marie. Il était à ce moment-là fort barbu et vieux, et pas marié. C'est en faisant, dit-il, quelque effort pour sauter que ses membres virils apparurent; et une chanson est encore en usage parmi les filles du pays par laquelle elles s'avertissent mutuellement de ne point faire de grandes enjambées de peur de devenir des garçons, comme Marie Germain. (Les Essais, I., 2009, p. I2I-122).

Dans cette perspective, la seule réalité qui soit, à propos du genre, est bien celle de l'action (ici au sens propre puisque c'est dans une action, le saut, que le genre surgit littéralement), de la situation, du moment, de l'immédiateté de l'expérience en somme, et toute tentative pour établir une forme d'enracinement se heurte au fait de passer à côté de la nature déterminante du genre, qui est d'être le fruit d'un rapport. D’où la promesse d'une impasse à tout essai d’appréhension du genre autre que pragmatique. Il est clair ici que le genre-visage s'intègre parfaitement dans l'ethnométhodologie de Harold Garfinkel ${ }^{12}$. Il me paraît même être un poste efficace pour observer les différences essentielles qui existent entre les approches du genre de Garfinkel et celles d'Erving Goffman (1973) ${ }^{13}$. C'est que

des expressions avant-gardistes du paradigme visage. L'on verra cependant par la suite que l'une de ses fondatrices, Judith Butler, a une position qui prête à confusion.

12 Le texte essentiel est ici l'analyse que livre l'auteur du cas Agnes, dans H. Garfinkel (1967, p. I16-185). Les problèmes, méthodologiques et éthiques, que suscite ce texte ont fait l'objet de nombreux débats: pour faire le point, lire M. Rogers (1992).

13 Pour un examen détaillé de ces écarts, on pourra désormais se reporter aux contributions de Danielle Chabaud-Rychter et Azadeh Kian dans D. Chabaud-Rychter, V. Descoutures, A.-M. Devreux, E. Varikas (2010). 
Garfinkel considère la réalité du genre exclusivement dans ses performances, ses actions (ce qui correspond au paradigme visage) tandis que Goffman maintient l'idée d'une «scène», d'un «décor» ou d'une «toile de fond» sur lesquels les acteurs déploient leur rôle d'homme ou de femme : le genre se fait masque. Dans le premier cas, le genre est agi; dans le second, il est porté. Au premier ne peut rendre justice qu'une thick description (pour reprendre la formule bien connue de $\mathrm{C}$. Geertz) qui restituerait, dans la terminologie des acteurs, tout l'environnement social, affectif, historique du geste qui dit le genre, tandis que le second pourrait se satisfaire d'une thin description qui documenterait une typologie dont les deux entrées principales resteraient le féminin et le masculin avec leurs caractères importés. C'est bien à cette typologie des figures féminines (et masculines en creux) quá permis d'introduire le dossier sur le compagnonnage.

Entre deux ouvres d'une très grande proximité, le questionnement du genre dynamisé par cette tension entre deux paradigmes divergents, le visage et le masque, introduit une ligne de rupture et permet de repenser à nouveaux frais et selon de nouveaux agencements les théories du genre dans les travaux sociologiques, historiques et anthropologiques.

Enfin, si le genre est d'abord une performance, s'il est fondamentalement un rapport (aux autres, à une situation) qui n’a d'autre épaisseur que l'action même qui l'actualise et l'environnement dans lequel il se déploie, alors surgit la possibilité d'une pluralité des genres dépassant de loin la bicatégorisation rigide, naturalisée, des sociétés occidentales modernes. La «multiplicité des voix sexuellement marquées » appelée de ses vœux par Jacques Derrida ouvre impertinemment le champ du genre dont la surface s'expose à des essais de cadastres inédits. L’alternative du masculin et du féminin cesse ici d'organiser absolument le genre (parce que le genre a fini d'être absolu), que ce soit sous l'espèce du tableau (soit l'un, soit l'autre) ou sous celle de la ligne (de l'un à l'autre). Le masculin et le féminin n'en viennent plus qu’à scintiller au milieu, et à égalité, d’une constellation de qualificatifs qui rendent le genre non pertinent ou trivial pour désigner la personne ${ }^{i 4}$. description impliquée par le genre-visage a chez eux un écho sous un autre lexique: «c'est en intensité, écrivent-ils, qu'il faut tout interpréter» (ibid., p. 186, mes italiques). 
Cette neutralisation du genre, qui reste dans la forme aboutie qui vient d'être décrite largement une vue de l'esprit, c'est-à-dire la déduction analytique du paradigme envisagé, se trouve, dans la réalité observée, dans un état nettement moins avancé mais qui, pour embryonnaire qu'il soit, ne laisse pas d'être hautement significatif. En effet, le pas à franchir qui mènerait à la neutralisation du genre consiste en la possibilité réservée au neutre d’exister comme catégorie des personnes. Non pas des hommes efféminées ou des femmes viriles, mais de véritables indéterminations qui restent en attente de signifiés pertinents. Or, ce neutre existe dans certaines sociétés qui, comme en Afrique de l'Ouest, rendent l'androgynie acceptable, sinon idéale, selon sa position générationnelle, notamment lorsque l'on fait partie des grands-parents (Peatrik A.-M, 1999). Il en est de même de l'existence d'une personne neutre comme le berdache des sociétés nord-américaines, qui n’est pas un «homme-qui-vit-à-la-manière-des-femmes » ou vice versa comme il en existe à Samoa (Tcherkézoff S., 2003) mais constitue bien un «troisième sexe» ainsi que l’a bien établi Bernard Saladin d’Anglure (1986) ${ }^{15}$. Enfin, les efforts réalisés par les anthropologues pour rassembler des faits attestant la présence, dans des langues et des cultures diverses, d'un «sexe relatif» (qui dépend de la personne qui parle et de celle à laquelle on s’adresse) vont dans ce sens (Alès C., Barraud C., 200I). Ainsi, pour reprendre un exemple cité par Irène Théry, chez les 'Aré’Aré des îles Salomon, le terme ahone sert à désigner le «germain de sexe opposé», donc une sœur si c'est un homme qui s'exprime, un frère s'il s’agit d'une femme (Théry I., 2009, p. 22).

D’ancien (je ne peux me résoudre à écrire «traditionnel» même avec des guillemets ici) qu'il était si l'on avait dû le classer dès l'abord, le paradigme visage se fait dès lors résolument postmoderne si l'on veut absolument sacrifier aux catégorisations en usage lorsque l'on veut situer les idées. Et l'on est d'autant plus en peine avec toute approche chronologique que, l'on aura l'occasion de le relever, l'autre paradigme que l'on va exposer à présent, celui du genre-masque, non seulement ne succède pas exactement à celui du visage mais trouve même, dans ses contours les plus extrêmes, une certaine proximité avec lui. Et, en effet, dans leurs marges respectives, les deux appréhensions du genre se trouvent être consonantes. 


\subsection{LE PARADIGME MASQUE \\ Le genre institué}

Le genre-masque est la perception du genre à laquelle, en sciences sociales au moins, nous sommes désormais le mieux rompus. Ce paradigme répond à celui du visage sur chacun des trois traits qui m’ont servi à caractériser ce dernier, à savoir la surface, la lecture et le monde unique et continu.

Dans l'ordre du masque, le genre ne s'expose plus exclusivement en surface. Il acquiert désormais une épaisseur nouvelle par l'enracinement de ses distinctions dans la physiologie. Il n'y a rien de plus étranger au genre-masque que les hermaphrodites ou les théories de l'androgyne premier qui ne contiennent pas au niveau élémentaire cette distinction considérée comme essentielle et fondamentale entre deux sexes. Mais cette croyance en cette différence première et décisive entre les sexes n'implique pas nécessairement l'assignation définitive à un rôle de genre. Justement, le paradigme du masque, et c'est là sa modernité, reconnaît, au moins dans un second temps (pour le compagnonnage, à partir des discussions sur la possibilité de femmes-compagnons), la possibilité à un individu d'adopter les attitudes et les comportements d'un sexe qui n'est pas physiologiquement le sien. Une distinction telle que celle opérée par Margaret Mead dans les années 1930 entre «sexe biologique» et «sexe social» à partir de son ethnographie du Sepik (Nouvelle-Guinée) relève incontestablement de ce paradigme (Mead M., I963, p. 31 I-320). Mieux, elle est de celles qui, pour les sciences sociales, ont contribué à l'ancrer profondément dans les esprits. D’une façon plus générale d'ailleurs, les approches constructivistes, et notamment au travers des gender studies, ont eu le souci de manifester la liberté des individus, et des femmes en particulier, en insistant sur le fait que le genre est un rôle, qu'il est donc socialement construit, et que sa correspondance avec le sexe dans lequel on est né n'est au mieux qu'une possibilité parmi d'autres, sans doute la plus commune parce que la plus facile. De cette proposition, l'on néglige souvent que, alors même qu'on cherche à démontrer que le genre se porte comme un vêtement, on l'inscrit dans le même temps dans le corps d'une façon bien plus profonde qu’auparavant. L'idée du «sexe social», celle du «moi» psychologique, celle de l'identité de genre a supposé, en négatif, la structuration du «sexe biologique», du «corps», de l’iden- 
tité de sexe. Autrement dit, en cherchant à dépasser et à abolir l'idée d'un destin de sexe, les sociétés occidentales modernes ont élevé à un tel niveau d'évidence la dualité des sexes qu'elles se sont empêchées de penser réellement, comme d'autres sociétés ont pu le faire, son dépassement. Ainsi, de même que le genre-visage ne se résume pas à une vision naturaliste du genre mais bien plutôt relationnelle et relativiste, le genre-masque ne constitue pas qu'une perspective socio-culturelle et constructiviste : il conduit à une naturalisation inédite du domaine.

Cela engage une nouvelle profondeur du genre. Le mode d’appréhension qu’il convient désormais de lui appliquer n’est plus celui de la lecture immédiate mais celui du déchiffrement. Dans le paradigme visage, le genre se lit. Mieux : il se voit. L'importance du regard rejoint l'idée que le corps est une scène permanente ignorant qu'il agit dans un théâtre. Sa réalité est unidimensionnelle: c'est celle de l'action dramatique. Dans le paradigme masque, l'aspect théâtral cesse d'être méconnu. De ce fait, l'on a supposé l'existence des décors, des rôles et, partant, de celle, distincte, des individus sous les masques, de la vie «réelle» au-delà de celle exposée sur les planches de la vie sociale. Mais comment y accéder puisqu'elle n'est pas montrée? Le regard et la lecture sont insuffisants; il faut ici l'enquête (sociologique, ethnologique, psychanalytique), l'analyse (structurale, interactionniste, cognitiviste). Autrement dit, il est nécessaire d’appliquer des instruments de déchiffrement qui au-delà de la vie jouée (dite, pensée) permettent de pénétrer la vie réelle (indicible, invisible, inconsciente). La mise au point et les avancées de la psychanalyse, au sein des problématiques du genre, rendent de ce fait parfaitement compte du paradigme masque (L. Irigaray, 1984).

Mieux, les recherches psychanalytiques comme celles de la psychologie cognitive ont porté loin ce paradigme. Elles ont conduit à inclure dans le masque le corps lui-même. Ce dernier cesse dès lors d'être le pourvoyeur essentiel de distinctions «naturelles». Le corps, désormais, est susceptible de masquer le sexe des individus. Qu'est-ce à dire? En fait, vers le milieu du Xx siècle, la «nature» a changé de lieux: elle était dans le corps, dans la physiologie; elle est désormais dans l'esprit et la chimie du cerveau. Et l'on est parfois «masqué» malgré soi, vivant dans un corps qui ne correspond pas à la nature de son esprit. Aussi, à l'inverse des hermaphrodites ou de l'androgyne premier, le phénomène des 
transsexuels, des changements de sexe d'une manière générale, s'inscrit parfaitement dans le paradigme masque ${ }^{16}$. Il ne s'agit en rien, les enquêtes sociologiques le montrent, d'une labilité du genre, mais au contraire de l'obéissance à une contrainte supérieure: l'exigence d'être de son «véritable genre». Et, en ce domaine plus quailleurs, il faut être capable d’avoir le «courage de la vérité $»^{17}$.

Ainsi, l'analyse, à tous les sens du terme, est fondamentalement l'outil et l'enjeu du décryptage du genre-masque. De même, les travaux de la microsociologie, ceux d'E. Goffman notamment (en opposition à ceux de H. Garfinkel encore une fois sous ce rapport), ont largement contribué à faire du masque la figure par laquelle le genre se doit d'être saisi.

Enfin, l'épaisseur de ce paradigme et la nécessité du déchiffrement qu’il impose conduisent à considérer que, pour le genre-masque, il existe plusieurs niveaux du monde, peut-être plusieurs mondes, hiérarchisés les uns par rapport aux autres (à l'inverse de la radicale équivalence du monde unique du paradigme visage). Sous le masque, il existe une vérité qui gît dans le corps, sa physiologie, sa génétique (ou dans le cerveau et sa chimie). Ce à quoi sattachent les tenants du masque est de démanteler le processus de mise au point et de port du genre comme rôle: l'on a affaire à une esthétique et à une génétique du genre. De ce point de vue, il s'agit notamment d'en examiner les contraintes. Quelle latitude avons-nous quand, nés dans un sexe, nous voulons ou décidons du genre qui sera le nôtre? La diversité des réponses à cette interrogation ne fait que traduire, au sein du paradigme masque, le niveau de contraintes «naturelles » auxquelles l'on estime que l'individu reste soumis. Parmi ceux qui le pensent élevé, on trouve notamment les tenants du droit et de la science qui, au XIX ${ }^{\mathrm{e}}$ siècle, ont développé et justifié l'appareil répressif dans le cadre d’un «dispositif nouveau de sexualité » donnant naissance aux «perversions », et finalement instituant des «hétérogénéités sexuelles» aboutissant au fait que,

16 Aussi, le cas de Marie-Germain relevé par Montaigne nous offre en vérité un beau cas de «polyphonie» sur le genre où se font entendre des voix, celles des villageois, celle du curé, celle de Montaigne, qui relèvent de paradigmes différents.

17 C'est le titre, hautement significatif et polysémique, de la dernière série de cours proposés par M. Foucault au Collège de France. 
par exemple, l'homosexuel devient véritablement une espèce selon l'expression de Michel Foucault (1976, p. 59). Au même degré, haut, de contraintes dans l'ordre du genre, se présentent également les partisans de la sociobiologie qui, sous le concert des attitudes et des rôles sociaux, s'appliquent à prêter l'oreille au «murmure intérieur chuchoté par nos gènes » et dont on voudrait qu'il soit la détermination dernière de tout comportement (Barash D., 1977, p. 293).

À l'autre pôle du masque, et comme à son extrémité qui rejoindrait là la frontière le séparant du visage, se distinguent les défenseurs d'un niveau faible, mais persistant, de contraintes imposées par le dimorphisme sexuel. Preuve de cette position frontière, l'on trouve ici certains représentants de la «théorie Queer» que l'on a par ailleurs globalement rattachée au paradigme visage. Ainsi, Judith Butler, outre qu’elle défend l'idée, toute «visagéenne», que le genre est performatif (Butler, 1992), refuse de penser qu'il puisse, tout doué de mobilité qu'il soit, être comparé à un vêtement que l'on changerait à sa guise car il existe, selon elle, un certain nombre d'éléments, dont le corps, qui sont contraignants (ibid., 1993).

L'existence de plusieurs mondes, la nécessité d’un décryptage et l'aspect épaissi du genre-masque l'ont rendu particulièrement accessible, en premier lieu, à ces entomologistes de la personne que sont, en Occident, les romanciers. Il n'est pas le lieu ici, et l'on n'en possède pas d'ailleurs les compétences, d'exposer les façons dont la littérature a pu se saisir de l'un ou l'autre de ces paradigmes $^{18}$. L'on notera simplement qu'elle constitue à nos yeux un poste privilégié d'observation concernant le saisissement du genre, et en particulier sous le régime du masque. Le cas de l'œuvre proustienne semble significatif de ce point de vue. On sait que l'écriture de Proust est démystificatrice en ce sens qu'elle cherche à faire tomber les masques des personnages et des situations dont d'infimes détails, repérés par la mémoire prodigieuse du narrateur, signalent la présence, la texture et les motivations. Il s'agit ainsi de noter ce qui relève de la «présentation de soi » d'une part, et d’autre part de la nature individuelle dont Proust montre qu'on lui prête beaucoup alors même qu'elle

18 Pour un bel et récent état des rapports entre littérature et sciences sociales, voir P. Baudorre, D. Rabaté, D. Viart (2007). 
représente peu dans la réalité. Ainsi, dans les premières pages de La Recherche, le narrateur, alors enfant, se voit sévèrement réprimandé par son père lorsqu'il se laisse submergé par ses émotions (Proust M., 1999, p. 38-39). Une plus grande maîtrise de celles-ci devrait, c'est le sentiment paternel, témoigner que l'enfant grandit et, en même temps, qu'il s'affirme dans son sexe. Mais le narrateur n'y parvient pas, ou pas suffisamment, et paradoxalement l'accès à un âge supérieur, comme une sorte de «puberté» spéciale selon les mots de Proust lui-même, se fera par la naturalisation de cette attitude. L'enfant sera considéré comme «nerveux». Ses larmes deviennent l'expression d'une nature particulière. Aussi, et c'est une des leçons de ce passage essentiel, la réduction biologique, c'est l'augmentation du sexe social. Je vois en cela le propre du masque.

Le message que nous délivre également ce dernier exemple est que la mise en mouvement du genre par l'un ou l'autre des paradigmes présentés ne se donne jamais mieux à voir que dans les situations critiques qui activent la conscience du genre et forcent les acteurs à l'appréhender pour lui-même. Ces moments de crise, que représentent notamment la période de l’adolescence et le passage à l'âge adulte, ou encore le phénomène des «invertis » comme l'on disait au XIX ${ }^{\mathrm{e}}$ siècle, et plus récemment les actes de changement de sexe, les efforts en faveur de la mixité (à l'école d'abord, au travail ensuite, etc.), forment tout un ensemble de gestes et de discours que l'on a pris l'habitude de synthétiser, et d'aplatir tout en même temps, dans l'expression de la «crise du genre », crise de la masculinité (Kimmel M., 1995 ; Levant R., 1997) ou de la domination masculine (Rauch A., 2006, p. I3) ${ }^{19}$, mais également crise de la féminité qui s'exprime notamment dans les développements du féminisme depuis ces balbutiements révolutionnaires jusqu’au mouvement queer récent.

Si ces situations critiques se donnent à voir plus particulièrement à partir de la fin du XVIII ${ }^{\mathrm{e}}$ siècle (parce que, après tout, l'on a toujours et partout passé à l'âge adulte), c'est que se développe à cette période un nouveau rapport à la vie, un rapport constitué davantage de responsabilité, de prise en charge personnelle

19 Pour faire le point sur les recherches concernant la masculinité et ses crises en Europe, voir I. Novikova (2004). 
(«faire quelque chose de sa vie»), une vie qui s'ouvre à plus de choix et d'options. L'existence cesse progressivement d'être simplement supportée ou admise une fois pour toutes; elle devient un véritable objet susceptible d'être manipulé, réorienté, éliminé également, sanctifié aussi. Le suicide égoïste (pour reprendre la terminologie de Durkheim) et le souci de la vie de tous les êtres (le fait qu'il existe toujours un ou des responsables d'une mort accidentelle et que celle-ci prend nécessairement le caractère d'un drame que l'empathie générale, dont la profondeur historique est sans doute maigre, fonde et élargit) sont des phénomènes proprement modernes. Il ne peut exister de «crise du genre » qu'à partir du moment où l'on considère que le genre relève de la responsabilité des individus. Le souci initiatique des compagnons (faire les hommes), l'attention portée à la place, c'est-à-dire au rôle des femmes, attestent le fait qu'ils ont conscience de leur responsabilité vis-à-vis de la production du genre. De la même façon, si l'adolescence devient un problème, ou plus précisément est considérée de plus en plus comme un âge singulier au XIX ${ }^{\mathrm{e}}$ siècle, c'est bien que les individus, dans l'ordre neuf de la vie-objet, ont nécessairement, et à certains moments plus qu'à d'autres, «la-vie-devant-soi » dont je pense qu'elle constitue l'une des caractéristiques fondamentales de l'existence moderne. Il s'agit tout à la fois d'une vie-objet (que l'on peut avoir sous le regard et que l'on peut embrasser : voir le développement des pratiques autobiographiques qui, en retour, renforce l'objectivation de la vie), d’une vie normée (l'existence a désormais des «patrons», des cadres idéaux [c'est la multiplication des «héros» qui s’industrialisent dans le «star system »] et non plus seulement des images singulières, précises et individualisées qu'incarnent les parents et qu'il s'agirait de reproduire), d'une vie choisie (le sentiment d'avoir élu les cadres adoptés et de pouvoir les congédier à tout moment; on en sait bien l'illusion) et dans laquelle, entre autres choix, celui de porter le genre (précisément comme un masque) est déterminant ${ }^{20}$.

20 Le paradigme de «la-vie-devant-soi» m’a été fourni par le roman éponyme de Romain Gary qui me paraît rassembler, dans le personnage de Madame Rosa, plusieurs des caractéristiques ici exposées concernant l'existence moderne. 


\section{BIBLIOGRAPHIE}

ADELL Nicolas, 2004, «Les sentiers de l'Orient. Initiation chez les compagnons du tour de France», Ethnologie française, vol. XXXIV, 3, p. 517-525.

-, 2006a, Des hommes de Devoir. Une ethnologie des compagnons du Tour de France (XVIII'-XXe siècle), thèse de doctorat de l'EHESS, Toulouse.

-, 2006b, «L'écriture de la sexualité. Les discours du sexe dans les autobiographies des compagnons Ménétra et Perdiguier», CLIO. Histoire, femmes, sociétés, 23, p. 293-209.

-, 2008, Des hommes de Devoir. Les compagnons du Tour de France (XVIII-XX siècle), Paris, Éditions de la Maison des sciences de l'homme.

ALĖS Catherine, BARRAUD Cécile (dir.), 200I, Sexe relatif ou sexe absolu?, Paris, Éditions de la Maison des sciences de l'homme.

ARTAUD Antonin, 2004a [1946], «Textes et lettres écrits à Rodez en 1946», in Euvres, Paris, Gallimard, «Quarto», p. 1051-1073.

-, 2004b [1948], «Pour en finir avec le jugement de Dieu», in Fuvres, Paris, Gallimard «Quarto», p. 1639-1654.

BARASH David P., 1977, Sociobiolgy and Behavior, New York, Elsevier.

BASTARD Laurent, 2004, «Les saints patrons des métiers du compagnonnage», Fragments d'histoire du Compagnonnage, vol. VI, p.7-59.

BAUDORRE Philippe, RABATE Dominique, VIART Dominique (dir.), 2007, Littérature et Sociologie, Pessac, Presses universitaires de Bordeaux III.

BUTLER Judith, 1992, Gender Trouble. Feminism and the Subversion of Identity, New York, Routledge.

-, 1993, Bodies that Matter. On the Discursive Limits of "Sex", Londres et New York, Routledge.

CAPDEVILA Luc, GODINEAU Dominique, 2004, «Éditorial», CLIO. Histoire, Femmes et Sociétés «Armées》, 20, p.5-14.

CARLINO Andrea, 1994, La Fabrica del corpo, Turin, Einaudi. 
CHABAUD-RYCHTER, Danielle, DESCOUTURES, Virginie, DEVREUX, AnneMarie, VARIKAS Eleni (dir.), 20I0, Sous les sciences sociales, le genre, Paris, La Découverte.

CONNAY Jean, 1909, Le Compagnonnage, son histoire, ses mystères, Paris, Union des charpentiers de la Seine.

DELEUZE Gilles, GUATTARI Félix, 1972, L’Anti-CEdipe, Paris, Éditions de Minuit. -, 1980, Mille plateaux, Paris, Éditions de Minuit.

DETIENNE Marcel, VERNANT Jean-Pierre, 1978 [1974], Les Ruses de l'intelligence. La mètis des Grecs, Paris, Flammarion, «Champs».

DREGER Alice D., 1998, Hermaphrodites and the Medical Invention of Sex, Cambridge, Harvard University Press.

FOUCAULT Michel, 1976, Histoire de la sexualité. I: La volonté de savoir, Paris, Gallimard.

GARFINKEL Harold, 1967, Studies in Ethnomethodology, Englewood Cliffs, NJ, Prentice-Hall.

GOFFMAN Erving, 1973, La Mise en scène de la vie quotidienne, Paris, Éditions de Minuit, 2 tomes.

GROSSMAN Evelyne, 2004, La Défiguration. Artaud - Beckett - Michaux, Paris, Éditions de Minuit.

HERDT Gilbert (dir.), 1994, Third Sex, Third Gender. Beyond the Sexual Dimorphism in Culture and History, New York, Zone Books.

IRIGARAY Luce, 1984, Éthique de la différence sexuelle, Paris, Éditions de Minuit.

KIMMEL Michael S. (dir.), 1995, The Politics of Manhood, Philadelphie, Temple University Press.

LAQUEUR Thomas, 1992, La Fabrique du sexe. Essai sur le corps et le sexe en Occident, Paris, Gallimard.

LEVANT Ronald F., 1997, "The masculinity crisis", Journal of Men's Studies, vol. V, p. 221-231.

MEAD Margaret, 1963 [1935], Mœurs et sexualité en Océanie, Paris, Plon, «Terre Humaine-Poche». 
MOLINIER Pascale, 2000, «Virilité défensive, masculinité créatrice», Travail, Genre et Sociétés, 3, p. 25-44.

MONTAIGNE Michel de, 2009, Les Essais, Paris, Gallimard, «Quarto».

NOVIKOVA Irina et al., 2004, "Men, Masculinities, and Europe", in Michael S. Kimmel, Jeff Hearn et Raewyn Connell (dir.), Handbook of Studies on Men and Masculinities, Thousand Oaks, Sage Publications, p. I4I-164.

PEATRIK Anne-Marie, 1999, La Vie à pas contés. Génération, âge et société dans les hautes terres du Kenya (Méru Tigania-Igembe), Nanterre, Société d'ethnologie.

PRUVOST Geneviève, 2007, Profession: policier, sexe: féminin, Paris, Éditions de la Maison des sciences de l'homme.

RAUCH André, 2006, Crise de l'identité masculine 1789-1914, Paris, Hachette, «Pluriel».

ROGERS Mary F., 1992, "They all Were Passing: Agnes, Garfinkel, and Company", Gender and Society, vol. VI, 2, p. 169-191.

RUBIN Gayle, 1998 [1975], «L'économie politique du sexe: transactions sur les femmes et systèmes sexe / genre», Les Cahiers du CEDREF, 7, p. 3-8I.

SALADIN D'ANGLURE Bernard, 1986, «Du fotus au chamane: la construction d'un "troisième sexe" inuit», Études/Inuit/Studies, vol. X, I-2, p. 25-113.

SOHN Anne-Marie, 2009, «Sois un homme!» La construction de la masculinité au XIXe siècle, Paris, Le Seuil.

STRATHERN Marilyn, 1988, The Gender of the Gift, Berkeley, University of California Press.

TCHERKEZOFF Serge, 2003, Faa samoa, une identité polynésienne, Paris, L'Harmattan.

THERY Irène, 2009, «Pour une anthropologie comparative de la distinction de sexe », in Pascale Bonnemère et Irène Théry (dir.), Ce que le genre fait aux personnes, Paris, Éditions de l'EHESS, p. 15-43. 
